\title{
Return-to-Play and Rehabilitation Protocols Following Isolated Meniscal Repair-A Systematic Review
}

\author{
Jordan W. Fried, B.M., Amit K. Manjunath, B.A., Eoghan T. Hurley, M.B., B.Ch., M.Ch., \\ Laith M. Jazrawi, M.D., Eric J. Strauss, M.D., and Kirk A. Campbell, M.D.
}

\begin{abstract}
Purpose: To systematically review the literature and assess the reported rehabilitation protocols, return-to-play guidelines, and reported rates of return-to-play after meniscal repair. Methods: MEDLINE, EMBASE, and the Cochrane Library were searched according to the Preferred Reporting Items for Systematic Reviews and Meta-Analyses (PRISMA) guidelines to identify studies on meniscal repair. Studies were included if return-to-play data and/or rehabilitation protocols were reported. The rate and timing of return-to-play was assessed. The rehabilitation protocols were documented, in addition to when to start range of motion (ROM), full ROM, partial weight-bearing (WB), and full WB. Results: Overall, 88 studies met our inclusion criteria. Thirteen studies, including 507 patients, cited a range of $71.2 \%$ to $100 \%$ of return-to-play, with $53.9 \%$ to $92.6 \%$ returning to the same/greater level, ranging between 3.3 and 10 months. There was considerable variability in the reported rehabilitation protocols, but the most frequently reported time to begin ROM exercises was within the first week (78.9\%) and full ROM at 6 weeks (33.3\%). Partial WB was typically begun during the first week (61.0\%), and full WB between the fourth and sixth week $(65.6 \%)$ postoperatively. Following surgery, time elapsed was the most commonly cited criteria for return-to-play (97.0\%), with 6 months being the most common time point applied $(46.9 \%)$. No study advised against returning to competitive or contact sports after meniscal repair. Conclusions: In conclusion, there was a high rate of return-to-play following meniscal repair, with $60 \%$ of patients returning to the same level of play. However, there was considerable diversity in the reported rehabilitation protocols and insufficient reporting on return-to-play criteria in the literature. This demonstrates the need for further research and formulation of an evidence-based consensus statement for this patient population. Level of Evidence: Level IV, systematic review of Level I to IV studies.
\end{abstract}

$\mathbf{M}$ eniscal tears are among the most common sports injuries managed by orthopaedic surgeons, with a reported annual incidence of 60 to 70 per 100,000 and an overall male predominance. ${ }^{1-4}$ Meniscal tears may be treated operatively or nonoperatively, with management decisions based on both patient factors (age, compliance, comorbidities) and tear

From the Department of Orthopaedic Surgery, Division of Sports Medicine, NYU Langone Health, New York, New York, U.S.A.

The authors report that they have no conflicts of interest in the authorship and publication of this article. Full ICMJE author disclosure forms are available for this article online, as supplementary material.

Received December 21, 2019; accepted July 29, 2020.

Address correspondence to Jordan W. Fried, B.M., Department of Orthopaedic Surgery, Division of Sports Medicine, NYU Langone Health, New York, NY 10010.E-mail: Jordanfried117@gmail.com

(C) 2020 THE AUTHORS. Published by Elsevier Inc. on behalf of the Arthroscopy Association of North America. This is an open access article under the CC BY-NC-ND license (http://creativecommons.org/licenses/by-nc-nd/4.0/).

2666-061X/191535

https://doi.org/10.1016/j.asmr.2020.07.023 characteristics. ${ }^{5}$ When surgery is determined necessary, meniscal repair is preferred over meniscectomy, as it preserves the load-reducing function of the meniscus and improves long-term clinical outcomes. ${ }^{6,7}$ Meniscal repairs are preferable for younger, healthier patients with reducible tears that are located within the red-red or red-white zones and characterized as longitudinal or horizontal. ${ }^{5}$

There is evidence that successful meniscal repair results in the prevention of future degenerative joint disease and preservation of more normal knee kinematics. $^{8-10}$ Prevention and preservation are crucial outcomes for patients, especially athletes, as they attempt recovery. However, while meniscal repair is the preferred technique when applicable, there is a lengthier rehabilitation and longer time before patients are capable of return-to-play to their desired sport. ${ }^{7,8,10}$ In addition, there is little information regarding how long patients should be rehabilitating, including the initiation of range of motion (ROM) exercises as well as 
the advancement of weight-bearing (WB) over time. Furthermore, there is little agreement for guidelines or criteria on how to allow for a safe return-to-play for athletes following isolated meniscal repair.

The purpose of this study was to systematically review the literature and assess the reported rehabilitation protocols, return-to-play guidelines, and reported rates of return-to-play after meniscal repair. Our hypothesis is there would be a high rate of return-to-play following meniscal repair but considerable variations in rehabilitation and return-to-play protocols are expected.

\section{Methods}

\section{Search Strategy and Study Selection}

To collect and compare the existing evidence relevant to rehabilitation protocols and return-to-play following isolated meniscal repair, a systematic review was performed according to the Preferred Reporting Items for Systematic Reviews and Meta-Analyses (PRISMA) guidelines. ${ }^{11}$ Clinical studies eligible for this review consisted of patients undergoing meniscal repair and reported a rehabilitation protocol and/or return-to-play data. For eligibility, studies must have been published in a peer-reviewed journal and available in full text. Studies considered ineligible for this review were those that used an open approach, used implants, and any repair with concomitant procedures that notably altered the rehabilitation protocol. Review articles, case reports, surgical technique articles, biomechanical studies, and non-English-language articles were not included.

Screening, eligibility, and inclusion were decided by 2 independent reviewers. The MEDLINE, EMBASE, and Cochrane Library databases were searched from their inception to October 2019 using the following algorithm: (menisc ${ }^{*}$ ) and (repair or rehab*). The titles and abstracts of the returned results were proofed according to the detailed eligibility criteria. Suitable studies were recognized, and the associated full text was reviewed. The reference lists of all relevant studies were screened for all articles that were not identified through the database search. Studies were included and accepted based on agreement of both independent reviewers. Any occurrences of disagreement were resolved through consultation with the senior author.

\section{Assessment of Level and Quality of Evidence}

The level of evidence (LOE) of the included studies was determined based on the criteria from The Oxford Centre for Evidence-Based Medicine. The methodologic quality of the return-to-play guidelines described in each study was measured based on the criteria outlined by Zaman et al. ${ }^{12}$ Every study was graded based on a scale from 0 to 4 , with 0 points demonstrating that the article did not include a return-to-play protocol and 4 points demonstrating a well-detailed return-to-play guideline. One point was awarded to every study for each of the following: inclusion of a rehabilitation protocol, specific measurements guiding safe return to activity, a time line for return to full sport or athletic activity, and any objective or subjective conditional criteria that must be met before return to full activity. Scoring discrepancies were discussed and settled through consultation with the senior author to reach an agreement.

\section{Data Extraction}

Two individual evaluators extracted data in duplicate from the included studies using a predesigned datacollection form. Screening was conducted to eliminate duplicate patients among studies. Demographic variables were composed of the total number of patients, sex ratio, patient age, and follow-up time. Variables affiliated with rehabilitation protocol included duration to achieve partial ROM, full ROM, partial WB, and full WB. Variables related to return-to-play included patient-reported timing of return to athletic activity, surgeon-proposed guidelines allowing athletes to return to full activity, and the percentage of patients returning to sport or play.

\section{Statistical Analysis}

Statistical analysis was performed using GraphPad Prism 7.05 (GraphPad Software, Inc., San Diego, CA). Descriptive statistics were calculated for all continuous and categorical variables. Continuous variables were reported as a weighted mean with an estimated standard deviation and categorical variables were reported as frequencies with percentages. Quality of return-toplay for each respective study was evaluated according to the Quality of the Return-to-Play guidelines outlined by Zaman et al. ${ }^{12}$

\section{Results}

In total, 6431 studies were initially identified. After extraction of duplicates, 3991 studies were further evaluated. After application of our inclusion/exclusion criteria, 88 studies reporting on 2776 knees were included in the final analysis (Fig 1)

\section{Demographics}

Overall, 88 clinical studies (LOE I: 4, LOE II: 17, LOE III: 33, LOE IV: 34) reporting return-to-play and/or rehabilitation guidelines with 2776 knees were included. There were 1163 male and 818 female patients, with a mean weighted age of 31.5 years (range $3-79$ ) followed up at a mean of 49.4 months (range 3-204).

\section{Rate and Time of Return-to-Play}

The rate of return-to-play ranged between $71.2 \%$ and $100 \%$ in 13 studies including 507 patients (Table 1). 
Fig 1. Preferred Reporting Items for Systematic Reviews and MetaAnalyses (PRISMA) study flowchart.
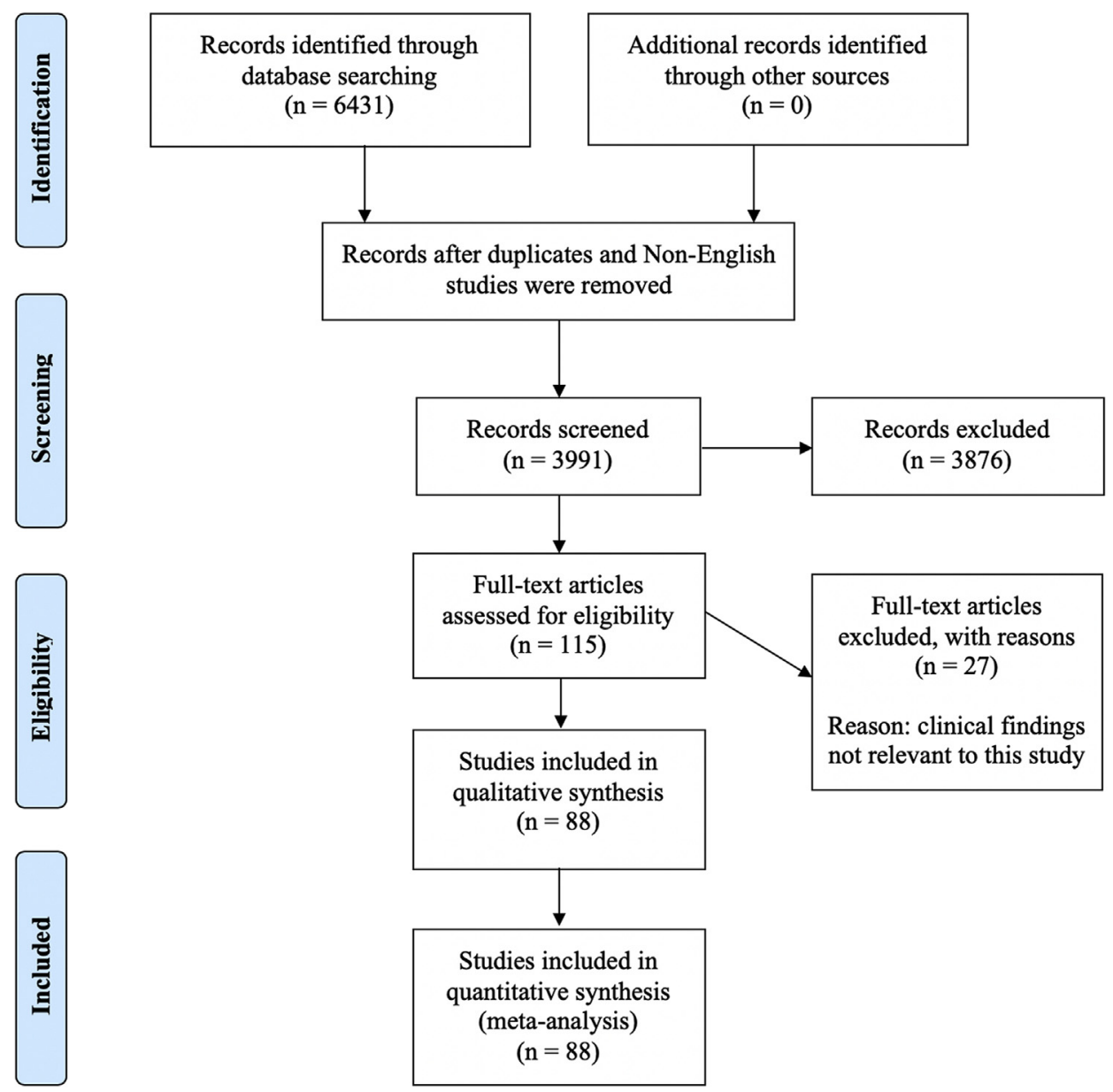

The rate of return-to-play at the same/greater level was reported ranged between $53.9 \%$ and $92.6 \%$ in 3 studies with 214 patients. The mean reported time of returnto-play ranged between 3.3 and 10 months in 11 studies with 409 patients (range 3.3-10).

\section{ROM Protocols}

The time to commencing ROM was reported in 76 studies (Table 2). The most commonly reported initiation of motion was within the first week $(78.9 \%)$, with $94.7 \%$ of studies reporting starting ROM in the first 2 weeks. The time to full ROM was reported in 63 studies. The most commonly reported time for allowing full

Table 1. Rate and Time of Return-to-Play

\begin{tabular}{ll}
\hline Rate of return-to-play & \\
& Range: $71.2 \%-100 \%$ \\
& 13 studies \\
$\begin{array}{l}\text { Rate of return-to-play at same/greater } \\
\text { level }\end{array}$ & $\begin{array}{l}\text { Range: } 53.9 \%-92.6 \% \\
3 \text { studies }\end{array}$ \\
Patient-reported return-to-play time & Range: $3.3-10$ \\
& 11 studies
\end{tabular}

ROM was 6 weeks postoperatively (33.3\%), followed by 4 weeks postoperatively $(25.3 \%)$. The time between commencing ROM and allowing for full ROM was reported in was reported in 62 studies, with 2 weeks between commencing ROM and full ROM being most commonly reported $(35.5 \%)$, followed by 4 weeks postoperatively $(27.4 \%)$.

\section{WB Protocols}

The time to partial WB was reported in 77 studies (Table 3). The most commonly reported initiation of partial WB was within the first week $(61.0 \%)$. The time to complete WB was reported in 67 studies. The most commonly reported time of commencing full WB was 4 and 6 weeks postoperatively $(32.8 \%)$. The time between partial and full WB was reported on in 64 studies, with 6 weeks between commencing partial and full WB being most commonly reported $(23.4 \%)$.

\section{Surgeon Guidelines and Criteria for Return-to-Play}

The surgeon guidelines for return-to-play were reported in 64 studies (Table 4). The most commonly reported time at which surgeons allowed return to full activity was 6 months postoperatively $(46.9 \%)$, but 
Table 2. Range of Motion Protocols

\begin{tabular}{lc}
\hline Range of motion & 76 studies \\
First week & $60(78.9 \%)$ \\
1 week & $1(1.3 \%)$ \\
2 weeks & $10(13.2 \%)$ \\
3 weeks & $4(5.3 \%)$ \\
4 weeks & $1(1.3 \%)$ \\
6 weeks & $1(1.3 \%)$ \\
Full range of motion & 63 studies \\
First week & $6(9.5 \%)$ \\
2 weeks & $6(9.5 \%)$ \\
3 weeks & $5(7.9 \%)$ \\
4 weeks & $16(25.4 \%)$ \\
5 weeks & $2(3.2 \%)$ \\
6 weeks & $21(33.3 \%)$ \\
8 weeks & $6(9.5 \%)$ \\
12 weeks & $1(1.6 \%)$ \\
Time between partial and full range of motion & 62 studies \\
Same time & $9(14.5 \%)$ \\
1 week & $1(1.6 \%)$ \\
2 weeks & $5(8.1 \%)$ \\
3 weeks & $4(6.5 \%)$ \\
4 weeks & $17(27.4 \%)$ \\
5 weeks & $2(3.2 \%)$ \\
6 weeks & $22(35.5 \%)$ \\
8 weeks & $2(3.2 \%)$ \\
\hline
\end{tabular}

one-half of the included studies allowed return before this time point. The mean quality of return-to-play criteria score was $1.3 \pm 0.8$. However, the conditional criteria and measurement for conditional criteria were under-reported in the majority of studies, $9.1 \%$ and $6.8 \%$ respectively. The most commonly reported conditional criteria was return of full ROM.

Table 3. Weight-Bearing Protocols

\begin{tabular}{lc}
\hline Partial weight-bearing & 77 studies \\
First week & $47(61.0 \%)$ \\
1 week & $1(1.3 \%)$ \\
2 weeks & $5(6.5 \%)$ \\
3 weeks & $3(3.9 \%)$ \\
4 weeks & $9(11.7 \%)$ \\
5 weeks & $1(1.3 \%)$ \\
6 weeks & $11(14.3 \%)$ \\
Full weight-bearing & 67 studies \\
First week & $9(13.4 \%)$ \\
2 weeks & $3(4.5 \%)$ \\
3 weeks & $4(6.0 \%)$ \\
4 weeks & $22(32.8 \%)$ \\
6 weeks & $22(32.8 \%)$ \\
7 weeks & $1(1.5 \%)$ \\
8 weeks & $5(7.5 \%)$ \\
10 weeks & $1(1.5 \%)$ \\
Time between partial and full weight-bearing & 64 studies \\
Same time & $14(21.9 \%)$ \\
1 week & $3(4.7 \%)$ \\
2 weeks & $12(18.8 \%)$ \\
3 weeks & $4(6.3 \%)$ \\
4 weeks & $14(21.9 \%)$ \\
6 weeks & $15(23.4 \%)$ \\
8 weeks & $1(1.6 \%)$ \\
10 weeks & $1(1.6 \%)$ \\
\hline
\end{tabular}

Table 4. Surgeon Guidelines and Criteria for Return-toPlay

\begin{tabular}{lc}
\hline Return-to-Play Criteria Score & $1.3 \pm 0.8$ \\
\hline Return-to-play timeline & 64 studies \\
3 months & $13(20.3 \%)$ \\
4 months & $15(23.4 \%)$ \\
5 months & $4(6.3 \%)$ \\
6 months & $30(46.9 \%)$ \\
9 months & $2(3.1 \%)$ \\
Criteria & 66 studies \\
Timeline & $64(97.0 \%)$ \\
Range of motion & $5(7.6 \%)$ \\
Strength & $3(4.5 \%)$ \\
Clinical decision & $3(4.5 \%)$ \\
Inflammation & $3(4.5 \%)$ \\
Pain & $2(3.0 \%)$ \\
Proprioception & $1(1.5 \%)$ \\
\hline
\end{tabular}

\section{Discussion}

The most significant finding from this systematic review of the literature was a high rate of return to sport following meniscal repair, with a large percentage of patients returning to the same level of play. Eberbach et al. ${ }^{13}$ reported good-to-excellent sports-specific outcomes following isolated meniscal repair, with a high return-to-play rate. However, there is significant heterogeneity in reported rehabilitation protocols and insufficient reporting in return-to-play criteria in the current literature. Furthermore, it remains vague in the literature when it is safe to return to play, with considerable variations with respect to when athletes could return.

Following all orthopaedic surgery procedures, return to complete preoperative function is a key and important desired outcome. Young, active patients place considerable emphasis on this outcome in their expectations following surgery. Our review showed that return-to-play and return to preoperative level was attainable at a high rate following meniscal repair, although there was a paucity of literature on return-toplay at the same level, and the results in studies reporting this were discouraging. Logan et al. ${ }^{14}$ assessed 42 elite athletes (professional, semi-professional, and amateurs competing at the state, national, or international level) and reported $81 \%$ were able to return to sports. In addition, they returned to play at a mean of 5.6 months with satisfactory results. Krych et al. ${ }^{15}$ reviewed 45 meniscal repair cases in the pediatric population and found all were able to return to play. However, only $62 \%$ of the 45 meniscal repair cases achieved clinical success and returned with no further issues. Those that were successfully repaired returned to sports at a mean of 5.5 months, compared with the unsuccessful group's return to sports at a mean of 4.5 months. These reported findings suggest that it is possible for athletes of varying ages to return to sports following meniscal repair. Despite these results, it is 
unknown if these athletes were able to maintain their activity level, or the impact of returning to sport has on the longevity of their meniscal repair. It is worth noting that the reported rates are comparable, if not better, than other common sports surgeries, including anterior cruciate ligament (ACL) reconstruction, shoulder stabilization, and medial patellofemoral ligament surgery. ${ }^{12,16-18}$

The vast majority of the studies assessed allowed for the onset of ROM exercises by postoperative week 1 . However, there was a discrepancy of when full ROM after meniscal repair was allowed, with most patients performing full ROM between the fourth and sixth weeks after surgery. It is not clear when it is safe to proceed with ROM exercises. Ideally, ROM should begin as early as possible to control the development of edema and muscle reactivation, preventing stiffness and limiting atrophy. ${ }^{19}$ Lind et al. ${ }^{20}$ compared free and restricted rehabilitation protocols beginning 2 to 3 days postoperatively and diverging within the first 2 weeks allowing the free-subgroup a ROM of $0-90^{\circ}$ and restricted-subgroup a ROM of $0-30^{\circ}$. This study concluded no difference in clinical outcomes, allowing safe return with a more liberal rehabilitation protocol. Wall et al. ${ }^{21}$ measured the biological effect of continuous passive motion of articular cartilage defects and found early resumption of ROM exercises in animal models proved to increase formation of hyaline repair tissue and the overall appearance of the articular cartilage, which is important to note as almost twothirds of knee arthroscopies have associated cartilage damage. Conversely, following meniscal repair, the knee may require immobilization initially to protect the repaired tissue from shearing and rotational stresses, which is the rationale behind our limiting WB at flexion angles greater than $90^{\circ}$ for the first 4 weeks following meniscal repair. ${ }^{22}$ Lucas et al. $^{23}$ retrospectively reviewed 17 pediatric patients and had them follow a rehabilitation protocol that included immobilizing the knee for 4 weeks, precluding excessive pivoting and flexion. Postoperative magnetic resonance imaging scans were taken for 10 patients and reported $60 \%$ good/excellent outcomes. Future research is needed to evaluate the proper balance between initiating ROM and protecting the repaired meniscus.

Full WB was allowed by weeks 4 to 6 in most of the studies evaluated, with significant variation in allowance of partial WB. A majority of patients were permitted partial WB by the end of week 1. Early WB following meniscus surgery has advantages, allowing early return to normal activities and encouraging a progression in activity and muscle strength. Still, there is concern that allowing full WB too rapidly may disrupt the repaired meniscus. In addition, if meniscal repair was performed with a concomitant procedure, the initiation of WB among other facets of rehabilitation was delayed with fear of setting back repair site healing. ${ }^{14}$

Significant variability was found in the return-to-play protocols and criteria after meniscal repair. Most studies solely cited time as the decisive factor allowing athletes to return-to-play, which varied between 3 and 6 months. Although approximate surgeon guidelines are critical benchmarks in allowing athletes to return to sport, other measures should be used. Quadriceps strength and ROM were evaluated and incorporated as part of the rehabilitation guidelines for a few of the included studies that implemented them into their return-to-play criteria. ${ }^{14,15,24-35}$ The use of arthroscopic findings to help determine to what level an athlete could return-to-play was not used in the included studies. However, Willinger et al. ${ }^{36}$ recorded magnetic resonance imaging scans at multiple postoperative time points and suggested future research correlating the images taken and a return-to-play guideline. There are few data currently available on meniscal healing at varying postoperative time points and its connection to return-to-play guidelines, which may influence a surgeon's decision making. No studies in our review advised against a return to competitive or high-impact sports following meniscal repair.

More research is necessary to determine which criteria must be met before athletes are safely able to return-to-play following meniscal repair. The American Academy of Orthopaedic Surgeons published a onepage checklist for specific patient-achieved goals, allowing a safe return-to-play following ACL reconstruction, and a comparable checklist could be composed for return-to-play following meniscal repair. ${ }^{37}$ Numerous criteria from the ACL checklist could be endorsed, including pain, full restoration of ROM, functional return of strength, consideration of local tissue healing and strength, and ability to perform sport-specific movement. The opportunity to assess and evaluate these criteria for a safe return-to-play has the chance to improve clinical outcomes and patient satisfaction, inviting further research opportunities.

\section{Limitations}

All systematic reviews are susceptible to limitations, including potential biases in the selected studies. Certain variables limit the conclusions made from this study, including the low level of evidence, retrospective design, and inconsistent reporting of return-to-play information. The type of meniscal tears was mixed in the majority of studies, making it difficult to perform any meaningful analysis comparing return-to-play rates, or assessing whether there was variation in rehabilitation protocols based on the tear configuration. Variation in rehabilitation protocol could also account for potential bias, influencing RTP rates. In addition, fixation technique was not analyzed and could have 
influenced return-to-play rates. The average rate and timing of return to sports may have been influenced by a particular sport and the level of competition. The variability in sports in the selected studies likely interferes with the ability to generalize the outcomes to all athletics. Although studies reporting return-to-play rates and rehabilitation criteria for meniscal repair with concomitant procedures were removed from inclusion, it was difficult to determine if concomitant procedures played a role on the return-to-play rate.

\section{Conclusions}

In conclusion, there was a high rate of return-to-play following meniscal repair, with $60 \%$ of patients returning to the same level of play. However, there was considerable diversity in the reported rehabilitation protocols, and insufficient reporting on return-to-play criteria in the literature. This demonstrates the need for further research and formulation of an evidencebased consensus statement for this patient population.

\section{References}

1. Beals CT, Magnussen RA, Graham WC, Flanigan DC. The prevalence of meniscal pathology in asymptomatic athletes. Sports Med 2016;46:1517-1524.

2. Nielsen AB, Yde J. Epidemiology of acute knee injuries: A prospective hospital investigation. J Trauma 1991;31: 1644-1648.

3. Greis PE, Bardana DD, Holmstrom MC, Burks RT. Meniscal injury: I. Basic science and evaluation. J Am Acad Orthop Surg 2002;10:168-176.

4. Gu YL, Wang YB. Treatment of meniscal injury: A current concept review. Chin J Traumatol 2010;13:370-376.

5. Mordecai SC, Al-Hadithy N, Ware HE, Gupte CM. Treatment of meniscal tears: An evidence based approach. World J Orthop 2014;5:233-241.

6. Paxton ES, Stock MV, Brophy RH. Meniscal repair versus partial meniscectomy: A systematic review comparing reoperation rates and clinical outcomes. Arthroscopy 2011;27:1275-1288.

7. Xu C, Zhao J. A meta-analysis comparing meniscal repair with meniscectomy in the treatment of meniscal tears: The more meniscus, the better outcome? Knee Surg Sports Traumatol Arthrosc 2015;23:164-170.

8. DeHaven KE, Lohrer WA, Lovelock JE. Long-term results of open meniscal repair. Am J Sports Med 1995;23: 524-530.

9. Johnson MJ, Lucas GL, Dusek JK, Henning CE. Isolated arthroscopic meniscal repair: A long-term outcome study (more than 10 years). Am J Sports Med 1999;27:44-49.

10. Stein T, Mehling AP, Welsch F, von Eisenhart-Rothe R, Jager A. Long-term outcome after arthroscopic meniscal repair versus arthroscopic partial meniscectomy for traumatic meniscal tears. Am J Sports Med 2010;38:1542-1548.

11. Moher D, Liberati A, Tetzlaff J, Altman DG, Group P. Reprint-preferred reporting items for systematic reviews and meta-analyses: The PRISMA statement. Phys Ther 2009;89:873-880.
12. Zaman S, White A, Shi WJ, Freedman KB, Dodson CC. Return-to-play guidelines after medial patellofemoral ligament surgery for recurrent patellar instability: A systematic review. Am J Sports Med 2018;46:2530-2539.

13. Eberbach H, Zwingmann J, Hohloch L, et al. Sport-specific outcomes after isolated meniscal repair: A systematic review. Knee Surg Sports Traumatol Arthrosc 2018;26: 762-771.

14. Logan M, Watts M, Owen J, Myers P. Meniscal repair in the elite athlete: Results of 45 repairs with a minimum 5year follow-up. Am J Sports Med 2009;37:1131-1134.

15. Krych AJ, McIntosh AL, Voll AE, Stuart MJ, Dahm DL. Arthroscopic repair of isolated meniscal tears in patients 18 years and younger. Am J Sports Med 2008;36: 1283-1289.

16. Fabricant PD, Chin CS, Conte S, Coleman SH, Pearle AD, Dines JS. Return to play after anterior cruciate ligament reconstruction in major league baseball athletes. Arthroscopy 2015;31:896-900.

17. Memon M, Kay J, Cadet ER, Shahsavar S, Simunovic N, Ayeni OR. Return to sport following arthroscopic Bankart repair: A systematic review. J Shoulder Elbow Surg 2018;27:1342-1347.

18. Ialenti MN, Mulvihill JD, Feinstein M, Zhang AL, Feeley BT. Return to play following shoulder stabilization: A systematic review and meta-analysis. Orthop J Sports Med 2017:5:2325967117726055.

19. Cinque ME, DePhillipo NN, Moatshe G, et al. Clinical outcomes of inside-out meniscal repair according to anatomic zone of the meniscal tear. Orthop J Sports Med 2019:7:2325967119860806.

20. Lind M, Nielsen T, Faunø P, Lund B, Christiansen SE. Free rehabilitation is safe after isolated meniscus repair: A prospective randomized trial comparing free with restricted rehabilitation regimens. Am J Sports Med 2013;41:2753-2758.

21. Wall A, Board T. The biological effect of continuous passive motion on the healing of full-thickness defects in articular cartilage. An experimental investigation in the rabbit. In: Banaszkiewicz P, Kader DF, eds. Classic papers in orthopaedics. London: Springer-Verlag, 2014;437-439.

22. Dowdy PA, Miniaci A, Arnoczky SP, Fowler PJ, Boughner DR. The effect of cast immobilization on meniscal healing. An experimental study in the dog. Am J Sports Med 1995:23:721-728.

23. Lucas G, Accadbled F, Violas P, Sales de Gauzy J, Knorr J. Isolated meniscal injuries in paediatric patients: Outcomes after arthroscopic repair. Orthop Traumatol Surg Res 2015;101:173-177.

24. Pujol N, Panarella L, Selmi TA, Neyret P, Fithian D, Beaufils P. Meniscal healing after meniscal repair: A CT arthrography assessment. Am J Sports Med 2008;36: 1489-1495.

25. Pujol N, Tardy N, Boisrenoult P, Beaufils P. Long-term outcomes of all-inside meniscal repair. Knee Surg Sports Traumatol Arthrosc 2015;23:219-224.

26. Nakayama H, Kanto R, Kambara S, et al. Clinical outcome of meniscus repair for isolated meniscus tear in athletes. Asia Pac J Sports Med Arthrosc Rehabil Technol 2017; 10:4-7. 
27. Tiftikçi U, Serbest S. Does the location of placement of meniscal sutures have a clinical effect in the all-inside repair of meniscocapsular tears? J Orthop Surg Res 2017;12:87.

28. Tïftikçï U, Serbest S. Repair of isolated horizontal meniscal tears with all-inside suture materials using the overlock method: Outcome study with a minimum 2-year followup. J Orthop Surg Res 2016;11:131.

29. Kaminski R, Kulinski K, Kozar-Kaminska K, Wasko MK, Langner M, Pomianowski S. Repair augmentation of unstable, complete vertical meniscal tears with bone marrow venting procedure: A prospective, randomized, double-blind, parallel-group, placebo-controlled study. Arthroscopy 2019;35:1500-1508.e1501.

30. Lons A, Boureau F, Drumez E, Pasquier G, Putman S. Does medial collateral ligament pie-crusting induce residual laxity in arthroscopic management of medial meniscus tears? A prospective study of 40 cases. Orthop Traumatol Surg Res 2018;104:707-711.

31. Haklar U, Kocaoglu B, Nalbantoglu U, Tuzuner T, Guven O. Arthroscopic repair of radial lateral menicus tear by double horizontal sutures with inside-outside technique. Knee 2008;15:355-359.
32. Asik M, Sen C, Erginsu M. Arthroscopic meniscal repair using T-fix. Knee Surg Sports Traumatol Arthrosc 2002;10: 284-288.

33. Alvarez-Diaz P, Alentorn-Geli E, Llobet F, Granados N, Steinbacher G, Cugat R. Return to play after all-inside meniscal repair in competitive football players: A minimum 5-year follow-up. Knee Surg Sports Traumatol Arthrosc 2016;24:1997-2001.

34. Barber FA, Johnson DH, Halbrecht JL. Arthroscopic meniscal repair using the BioStinger. Arthroscopy 2005;21: 744-750.

35. Barber FA, Schroeder FA, Oro FB, Beavis RC. FasT-Fix meniscal repair: Mid-term results. Arthroscopy 2008;24: 1342-1348.

36. Willinger L, Herbst E, Diermeier T, et al. High short-term return to sports rate despite an ongoing healing process after acute meniscus repair in young athletes. Knee Surg Sports Traumatol Arthrosc 2019;27:215-222.

37. AAOS. ACL Reconstruction patient return to play checklist. https://www.aaos.org/globalassets/quality-andpractice-resources/patient-safety/clinician-checklists/aaosacl-return-to-play-checklist.pdf. Accessed July 29, 2020. 